\title{
Impact of COVID-19 pandemic on happiness and stress: comparison of preclinical and clinical medical students
}

\author{
Suwicha Kaewsiri Isaradisaikul', Pichcha Thansuwonnont ${ }^{2}$ and Phatsaworn Sangthongluan ${ }^{1}$ \\ ${ }^{1}$ Department of Otolaryngology, Faculty of Medicine, Chiang Mai University and ${ }^{2}$ Faculty of Medicine, Chiang \\ Mai University, Chiang Mai, Thailand
}

Purpose: To compare differences in happiness and stress and related factors between pre-clinical and clinical year medical students during the coronavirus disease 2019 (COVID-19) pandemic.

Methods: A cross-sectional study was conducted in the Faculty of Medicine, Chiang Mai University, Thailand. All undergraduate medical students were requested to voluntarily respond to an electronic survey. Demographic data, related factors of happiness and stress, scores from the Thai version of the Oxford Happiness Questionnaire (Thai-OHQ), and Thai Stress Questionnaire (Thai-ST5) were collected.

Results: There were 369 responses, $64.8 \%$ from preclinical students and $35.2 \%$ responses from clinical students, and $53.9 \%$ were women. The mean age of the participants was $20.62 \pm 1.81$ years. The most frequent platforms that the students used to track COVID-19 information were Facebook $43.9 \%$ and Twitter $43.4 \%$. Both groups had a low level of stress. No difference was found in the Thai-OHO score $(p=0.323)$ and the Thai-ST5 score $(p=0.278)$. With multivariable analysis, two factors significantly related to the happier students included higher health satisfaction scores $(p<0.001)$ and maintaining an exercise program during the COVID-19 pandemic $(p=0.015)$.

Conclusion: There was no difference in the happiness and stress levels between the two groups during the first outbreak of COVID-19 in Thailand. To increase happiness, promoting awareness of health satisfaction and regularity of exercise for the medical students should be initiated. To direct the information during a disease outbreak such as the COVID-19 pandemic, Facebook, and Twitter are the primary platforms to use.

Key Words: Medical students, Happiness, Stress, COVID-19 pandemic, Oxford happiness questionnaire

\section{Introduction}

The coronavirus disease 2019 (COVID-19) pandemic has caused intense effects on all aspects of society, mental health, and physical health [1,2]. The psychological effect of the COVID-19 pandemic has been associated with distress, anxiety, fear of contagion, and depression in the general population and among health care professionals [2]. Beginning with the first report of cluster pneumonia in Wuhan, China, in late December 2019, COVID-19 rapidly spread [3]. Thailand was the second country after China to report the first case of COVID-19 infection on January 15, 2020 [4]. The World Health Organization reported over one million worldwide confirmed cases of COVID-19 by April 4, 2020.
Received: February 25, 2021 • Revised: April 16, 2021 • Accepted: April 19, 2021 Corresponding Author: Suwicha Kaewsiri Isaradisaikul (https://orcid.org/0000-0003-1332-9940) Department of Otolaryngology, Faculty of Medicine, Chiang Mai University, 110 Intawaroros Road, Sriphum, Meuang, Chiang Mai, Thailand

Tel: +66.53935562 Fax: +66.53935564 email: suwicha.kaewsiri@cmu.ac.th
Korean J Med Educ 2021 Jun; 33(2): 75-85

https://doi.org/10.3946/kjme.2021.189

eISSN: 2005-7288

(C) The Korean Society of Medical Education. All rights reserved. This is an open-access article distributed under the terms of the Creative Commons Attribution Non-Commercial License (http:// creativecommons.org/licenses/by-nc/3.0/), which permits unrestricted non-commercial use, distribution, and reproduction in any medium, provided the original work is properly cited. 
Since then, COVID-19 has been noted as a "pandemic" [5]. A nationwide nighttime curfew in Thailand was in effect from April 3, 2020, through June 15, 2020, during the first outbreak [6]. The COVID-19 pandemic affected all healthcare personnel including medical students creating increased stress because of the mode of transmission, tremendous fear, and implementation of infection control protocols [7].

Medical students are among the top of the academically high-performance university students. Between 50\%-60\% of medical students had stress and burnout [8]. With a higher responsibility in the health-related field and with a higher family and social expectations, higher stress and exhaustion were expected. However, $60.8 \%-85.8 \%$ of medical students were happy [9-11]. Their rates of happiness and stress were higher than the general student population $[8,10]$. Studies regarding the psychological health of medical students primarily reported negative aspects that included depression and anxiety. Few studies investigated happiness [10-12] and rarely reported the impact of the COVID-19 pandemic on the happiness and stress of medical students.

Measurements of happiness which have been widely studied in international settings usually included the subjective wellbeing and satisfaction in life [13]. The Oxford Happiness Questionnaire (OHQ), developed by Hills and Argyle [14] in 2002, is one of the most widely used questionnaires for studies of happiness. The summation of all items is an overall measure of happiness, with high scores indicating greater happiness [14]. The Thai version of OHQ (Thai-OHQ) was translated and validated by Phoomchan [15]. The Thai Stress Questionnaire (Thai-ST5) was developed by Silpakit [16] and had acceptable concurrent validity with the Hospital Anxiety and Depression Scale. During the COVID-19 pandemic, the website of the Department of
Mental Health, Ministry of Public Health of Thailand publicized the Thai-ST5 as one of the assessment tools. The authors received permission for the use of the Thai-OHQ and Thai Stress Assessment Questionnaire.

The impact of COVID-19 on medical education was found to affect both the preclinical and clinical learning environments [17]. Changes included replacement of in-person classes to an online mode, cancellation of clinical clerkships, elective surgical procedures, and routine non-emergency appointments, and limitations of the role of students in the clinical environment to minimize personal interactions, decrease the risk of exposure for medical students, and conserve the use of personal protective equipment $[17,18]$.

The lack of adequate knowledge of medical students could cause exaggeration of the situation and an increase in stress and anxiety [19]. Psychological stress from the outbreak possibly affects the learning and overall psychological health of medical students [7].

Faculty of Medicine, Chiang Mai University (CMU), the third largest Thai medical school in Northern Thailand was established in 1959. The curriculum structure of medical schools in Thailand is a 6-year integrated course that recruits students from high schools. The course includes the first, second, and third years of preclinical courses and three additional years of clinical clerkships. During the first COVID-19 pandemic, CMU medical school switched all in-class academic activities to an online mode, delayed the start of the semester, rearranged and limited the on-call and clinical practice schedule to minimize exposure risks of the medical students. After the lockdown relaxed, the students returned to medical school with new-normal safety measures. The new-emerging phase of COVID-19 outbreaks in Thailand started in December 2020.

This study aimed to determine the differences in happiness and stress and their related factors between 
pre-clinical (first through the third year) and clinical (fourth through the sixth year) CMU medical students during the first outbreak of COVID-19 in Thailand. Comparison of the happiness and stress between the two groups were in authors' interesting due to differences of (1) role of students in the clinical environment, (2) minimum requirement in the curriculum, and (3) depth of medical knowledge. The study protocol was approved by the Research Ethics Committee of the Faculty of Medicine, Chiang Mai University (study code: ENT2563-07662, research ID: 7662). Results may assist the Faculty with strategic planning for the next outbreak, guide medical educators for improvement of the curriculum design, or apply to other medical schools to minimize the impact of COVID-19 on happiness and stress.

\section{Methods}

This cross-sectional study was conducted after approval of the Research Ethics Committee. All current undergraduate medical students in the Faculty of Medicine, CMU, age 18 years or older, and primarily used the spoken Thai language were requested to voluntarily respond to an electronic survey. The students were informed about the research project and asked to complete the questionnaire through smartphone LINE application groups and the Student Affairs Office.

The questionnaire was generated with Google Forms. After acceptance of the informed consent, the student then accessed the three sections of the questionnaire: (1) personal information and outcome-related factors, (2) stress questionnaire, and (3) happiness questionnaire. The stress questionnaire included five items with a 4-point Likert-type scale of the Thai-ST5. Total scores of Thai-ST5 at 0-4, 5-7, 8-9, and 10-15 indicate mild, moderate, high, and severe stress level, respectively. The happiness questionnaires included (1) a happiness score which is a one-question with 0 (minimum)-10 (maximum) scale during with and without the COVID-19 pandemic and (2) 29 items with a 4-point Likert-type scale of the Thai-OHQ. The test for the Thai-OHQ validity showed Cronbach's $\alpha=0.8607$. About $10-15$ minutes were required to complete the questionnaire. The participants were required to answer all questions except the question about income and liabilities, under the suggestion of the Ethics Committee. The data was collected from November 19, 2020, to December 7, 2020. The collection had to stop before a new-emerging phase of COVID-19 outbreaks in Thailand.

The data were analyzed using the Stata ver. 16.0 software (Stata Corp., College Station, USA). Descriptive statistics were used to present demographic data. Differences in variables between the two groups were compared using the chi-square test, independent t-test, or Mann-Whitney U-test as appropriate. Factors related to happiness and stress according to previous reports and all variables that showed significant differences between the preclinical and clinical years (with its $\mathrm{p}$-value $<0.05$ ) were adjusted using linear regression to identify the differences in the outcomes. A p-value $<0.05$ was statistically significant.

\section{Results}

Of the total of 1,445 medical students in the 2020 academic year, 393 responses were returned. Twenty duplicate responses and four unacceptance of the informed consent were excluded. The response rate was $25.5 \%$ (369 of 1,445). Two hundred thirty-nine (64.8\%) responses from preclinical students and 130 (35.2\%) clinical students were received. The responses were 
returned from the medical students in the first year (26.0\%), second year (26.8\%), third year (11.9\%), fourth year (14.9\%), fifth year (7.9\%), and sixth year (12.5\%). The highest rate of response was among the second-year students (41.8\% of the second-year students), the lowest response was among the fifth-year students $(12.2 \%$ of the fifth-year students). Women represented 53.9\% of the responses. The mean age of participants was $20.62 \pm 1.81$ years (minimum=18, maximum=25).

The demographic characteristics, lifestyle, student and family income, and liabilities during the COVID-19 pandemic between preclinical and clinical medical students are shown in Tables 1 and 2. Factors that showed a significant difference between preclinical and clinical students were health satisfaction score, grade point average (GPA), numbers of members in the accommodation, impact of academic activity changing to the learning outcome, spending time with their family or returning to their hometown, average of their monthly income, and their liabilities.

During the COVID-19 pandemic, the most frequent activities that the student used to relieve their stress were movies or music $33.6 \%$, online media $32 \%$, sleeping $12.7 \%$, sports $6.5 \%$, musical instruments $6.5 \%$, reading books $4.3 \%$, games $3.5 \%$, and others $0.8 \%$. The most frequent resources that the students used to receive the information about COVID-19 were from news channels $54.7 \%$, relatives/friends $18.4 \%$, social media $12.2 \%$, internet $7.9 \%$, government organizations $6.5 \%$, and others $0.3 \%$. The most frequent platform that the students used to track information about COVID-19 were from Facebook 43.9\%, Twitter 43.4\%, TV 6.2\%, Instagram 3.5\%, LINE application 1.9\%, TV online $0.5 \%$, newspaper $0 \%$, and others $0.5 \%$.

The Happiness score, Thai-OHQ score, and Thai-ST5 score between preclinical and clinical medical students are shown in Table 3. Both groups had a low level of stress. No difference was found in the Thai-ST5 score

Table 1. Demographic Characteristics between Preclinical and Clinical Medical Students

\begin{tabular}{|c|c|c|c|c|}
\hline Characteristic & Category & Preclinical ( $=239$ ) & Clinical $(n=130)$ & $\mathrm{p}$-value \\
\hline Age (yr) & Mean (min-max) & $19(18-24)$ & $23(21-25)$ & $<0.0001^{\text {al }}$ \\
\hline \multirow[t]{3}{*}{ Gender } & Male & $103(43.10)$ & $54(41.54)$ & $0.939^{b /}$ \\
\hline & Female & $128(53.56)$ & $71(54.62)$ & \\
\hline & Alternative sex & 8 (3.35) & $5(3.85)$ & \\
\hline Body mass index $\left(\mathrm{kg} / \mathrm{m}^{2}\right)$ & Mean \pm SD $(95 \%$ CI) & $21.94 \pm 3.69(21.47-22.41)$ & $21.55 \pm 3.10$ (21.01-22.09) & $0.313^{c l}$ \\
\hline \multirow[t]{2}{*}{ No. of sibling } & 1 & $44(18.41)$ & 23 (17.69) & $0.864^{b /}$ \\
\hline & $>1$ & 195 (81.59) & 107 (82.31) & \\
\hline \multirow[t]{2}{*}{ Underlying disease } & Present & $50(20.92)$ & $31(23.85)$ & $0.517^{b l}$ \\
\hline & Absent & 189 (79.08) & $99(76.15)$ & \\
\hline Health satisfaction score ${ }^{\mathrm{d})}$ & Mean \pm SD $(95 \%$ CI) & $3.68 \pm 0.77(3.58-3.78)$ & $3.88 \pm 0.74(3.75-4.00)$ & $0.019^{\mathrm{cl}_{1, *}}$ \\
\hline \multirow[t]{2}{*}{ Self-decision to study in the MD program } & Yes & 218 (91.21) & 117 (90.00) & $0.700^{b l}$ \\
\hline & No & $21(8.79)$ & $13(10.00)$ & \\
\hline Grade point average & Mean \pm SD $(95 \%$ CI) & $3.49 \pm 0.42(3.44-3.54)$ & $3.23 \pm 0.39(3.16-3.29)$ & $<0.0001^{c c_{1, *}}$ \\
\hline \multirow[t]{2}{*}{ Having own vehicle } & Yes & $150(62.76)$ & $92(70.77)$ & $0.122^{b l}$ \\
\hline & No & 89 (37.24) & 38 (29.23) & \\
\hline \multirow[t]{2}{*}{ Staying in the Faculty dorm } & Yes & $122(51.05)$ & $73(56.15)$ & $0.348^{b l}$ \\
\hline & No & $117(48.95)$ & $57(43.85)$ & \\
\hline No. of members in the accommodation & Median (min-max) & $2(1-8)$ & $2(1-7)$ & $0.044^{\mathrm{a}, *}$ \\
\hline
\end{tabular}

Data are presented as mean (min-max), number (\%), mean \pm SD $(95 \%$ CI), or median (min-max), unless otherwise stated.

SD: Standard deviation, Cl: Confidence interval, MD program: Doctor of Medicine program.

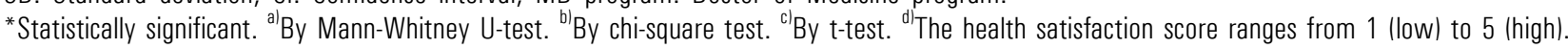




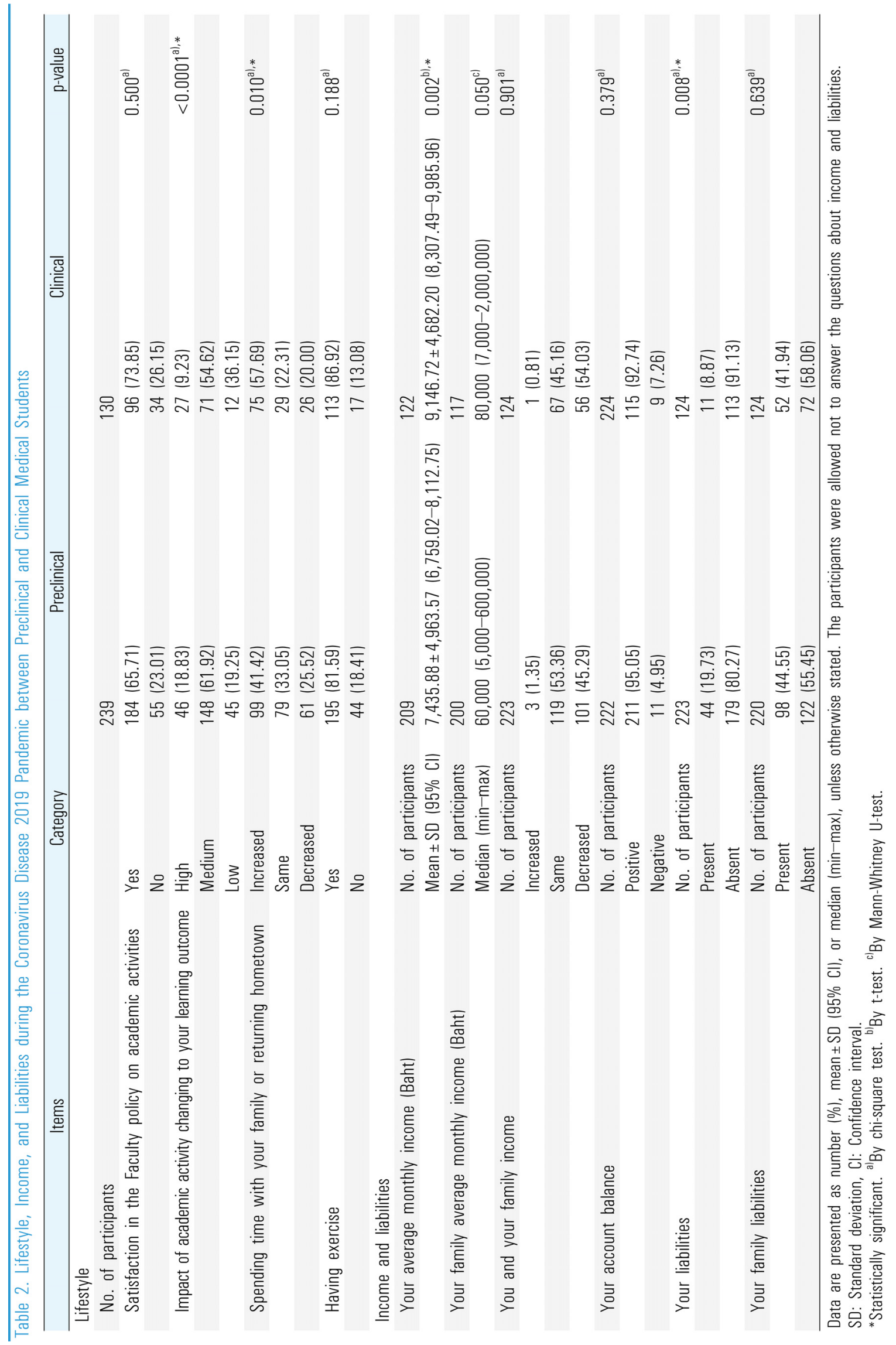


Table 3. Happiness, Thai-0HQ, and Thai-ST5 Scores between Preclinical and Clinical Medical Students

\begin{tabular}{lccc}
\hline \multicolumn{1}{c}{ Items } & \multicolumn{1}{c}{ Preclinical $(\mathrm{n}=239)$} & \multicolumn{1}{c}{ Clinical $(\mathrm{n}=130)$} & $\mathrm{p}$-value \\
\hline Happiness score during no COVID-19 pandemic & $7.71 \pm 1.55(7.51-7.91)$ & $7.5 \pm 1.45(7.25-7.75)$ & $0.2012^{\mathrm{al}}$ \\
Happiness score during the COVID-19 pandemic & $6.02 \pm 1.97(5.77-6.28)$ & $6.7 \pm 1.73(6.39-7.00)$ & $0.001^{\mathrm{a}, * *}$ \\
Thai-OHO score during the COVID-19 pandemic & $25.15 \pm 99.69(68.05-71.93)$ & $73.68 \pm 14.31(71.20-76.17)$ & $0.024^{b)_{\text {, }}}$ \\
Thai-ST5 score & $4(0-14)$ & $4(0-12)$ & $0.278^{\text {c) }}$ \\
\hline
\end{tabular}

Data are presented as mean \pm standard deviation (95\% confidence interval) or median (min-max), unless otherwise stated.

Thai-OHQ: Thai version of Oxford Happiness Questionnaire, Thai-ST5: Thai Stress Assessment Questionnaire, COVID-19: Coronavirus disease 2019.

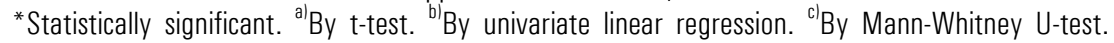

Table 4. Thai-OHO Score during the COVID-19 Pandemic between Preclinical and Clinical Medical Students after Adjusted Related Factors ${ }^{\text {al }}$

\begin{tabular}{cccc}
\hline Item & Preclinical $(\mathrm{n}=239)$ & Clinical $(\mathrm{n}=130)$ & $\mathrm{p}$-value \\
\hline Thai-OHO score & $70.76(68.08-73.43)$ & $73.70(69.71-77.69)$ & $0.323^{\mathrm{b})}$ \\
\hline
\end{tabular}

Data are presented as median (min-max).

Thai-OHQ: Thai Version of Oxford Happiness Questionnaire, COVID-19: Coronavirus disease 2019.

${ }^{\text {a) }}$ Adjusted factors included (1) demographic characteristics lage, gender, body mass index, numbers of sibling, underlying disease, health satisfaction score, grade point average, having own vehicle, staying in the Faculty dorm, and numbers of members in the accommodation), (2) lifestyle during the COVID-19 pandemic limpact of academic activity changing to the learning outcome, spending time with family or return to hometown, and having exercise), and (3) income and liabilities (average monthly income and liabilities of students and family, and account balance. ${ }^{b / B y}$ a multivariable linear regression.

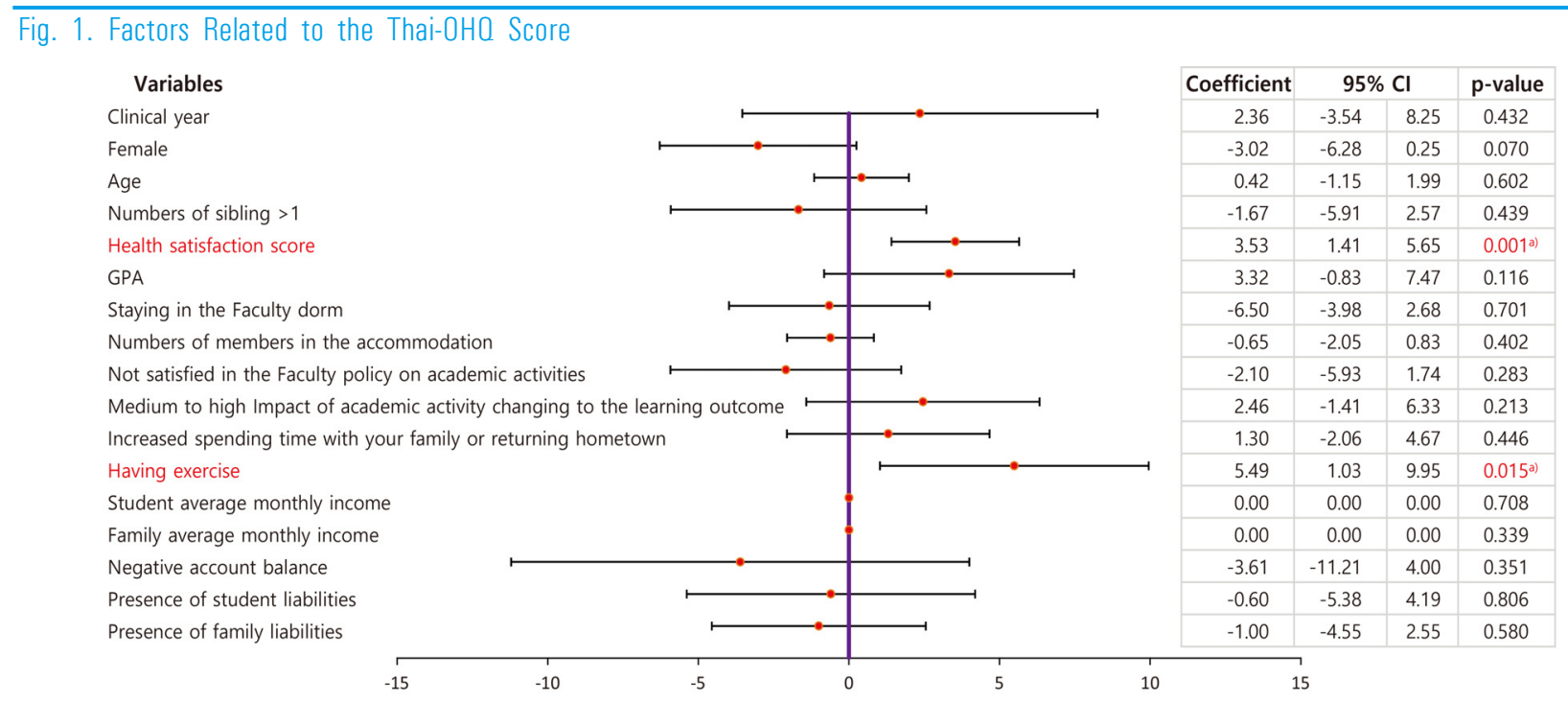

Thai-OHQ: Thai Version of Oxford Happiness Questionnaire, Cl: Confidence interval, GPA: Grade point average. ${ }^{\text {al }}$ Statistically significant between preclinical and clinical students.

during the COVID-19 pandemic and happiness score during no COVID-19 pandemic. During the COVID-19 pandemic, the happiness score and Thai-OHQ score of the preclinical students were significantly lower than of the clinical students. However, the Thai-OHQ score between the two groups and showed no differences $(\mathrm{p}=0.323)$ after analysis with multivariable linear regression to adjust related factors as shown in Table 4.
With multivariable analysis, two factors significantly related to the happier students (higher Thai-OHQ score) included higher health satisfaction score and having exercise during the COVID-19 pandemic, as shown in Fig. 1. Because of the non-normal distribution of the stress level or Thai-ST5 score, no multivariable analysis was performed. 


\section{Discussion}

Providing authentic patient experiences for medical students as a key component of medical education is more challenging in risk situations such as the COVID-19 pandemic [17]. Although 19.3\% of medical students did not regard the COVID-19 pandemic as a severe public health problem [20], 35\% of medical students preferred not to return to the clinical education setting, due to concerns about the risk of infection [21].

To graduate with a Doctor of Medicine (MD) degree, medical students are required to meet the minimum requirement in the curriculum and have proper milestone achievements with various supports. Evidence-based information about factors affecting any aspects of medical students' needs while studying in the MD program should be explored. Happiness and stress are two of these interesting factors. The great changes from the COVID-19 pandemic brought about many factors that affected the happiness and stress of medical students.

Many factors related to the happiness of medical students in previous reports included own health satisfaction, self-decision to study in the $\mathrm{MD}$ program, having own vehicle, prior perception of medical student's life-style [10], gender, younger siblings [9], frequency of exercise or level of physical activity, underlying disease [12], and body mass index [22]. These factors were derived from previous studies prior to the impact of the COVID-19 pandemic. In this study, two factors (health satisfaction score and having exercise during the COVID-19 pandemic) were significantly related to happiness.

Significantly higher student average monthly income and a higher rate of absent liabilities were found in the CMU clinical students than in the preclinical medical students. According to Fig. 1, although no significant effect of gender, account balance and presence of students' and families' liability on the happiness or Thai-OHQ scores of the CMU medical students, female CMU medical students or students who had negative account balance had a trend to have lower Thai OHQ scores. Higher scores of happiness of the second-year medical students in India were significantly related to the male gender [9]. Female medical students in Saudi Arabia also had a significantly higher mean stress level than males [7]. A lower family income was related to Turkish medical student anxiety and stress [19].

The health satisfaction score of the CMU clinical year medical students $(3.88 \pm 0.74)$ was significantly higher than that of preclinical students $(3.68 \pm 0.77)$. A study to confirm its clinical significance is suggested. However, the higher health satisfaction score showed a significant relationship to the happiness or Thai-OHQ score. The GPA of the CMU preclinical medical students was significantly higher than that of the clinical students. The higher expectation to maintain a high GPA under online lessons was a possible challenge. Although no significant relationship to the happiness or Thai-OHQ scores, the students with higher GPA trended to have more happiness.

The most frequent platform that the CMU medical students used to follow the information about COVID-19 was from social media (87.3\%) higher than of Turkish medical students (50.8\%). Of these, $82.0 \%$ of Turkish students reported distrusting information/messages from social media and $52.4 \%$ reported feeling mentally unwell as they obtained unfiltered information [20].

Most of the CMU students $(65.71 \%$ of preclinical, $73.85 \%$ of clinical students) were satisfied with the Faculty policy on academic activities during the COVID-19 pandemic, similar to the satisfaction rate of the third-year clerkship medical students in South Korea 
(75\%) [23]. The rate was much higher than the satisfaction rate of clinical year medical students in Jordan [24] (26.8\%). About 68.1\% of Turkish medical students wished to receive distance learning. A higher rate of preferred distance learning for Turkish medical students was found in the sixth-year medical students (86.5\%) than in the first-year medical students (61.9\%) [20]. Challenges of the distance e-learning in Jordan mostly resulted from poor internet coverage (69.1\%), limitations in internet data packages (38.1\%), and variation in educational platforms (38.1\%) [24]. Students' and instructors' experiences affected the satisfaction of distance learning. Up to $77.1 \%$ of the Jordan medical students had never enrolled in distance learning related to the university curriculum. Before the COVID-19 pandemic, the CMU medical school was beginning to reduce in-class lecture times. Transformed modes of academic delivery based on the remodeling of the $\mathrm{MD}$ program curriculum in 2015 which emphasized teamfacilitated problem-based and self-directed learning. This could have resulted in a higher rate of satisfaction.

The CMU preclinical students $(80.75 \%)$ reported the impact of academic activities that changed their learning outcomes from medium to a high level, which was significantly greater than of clinical student (63.85\%). The CMU clinical year medical students (57.69\%) had a significantly higher chance of spending time with their family or returning hometown than preclinical students (41.42\%). Possible explanations include higher numbers of preclinical in-class lecture changed to online mode compared to clinical practice in the wards. Cancellation of clinical practice offered the clinical year medical students more time for self-learning, returning home for a family reunion, and less risk of COVID-19 exposure in clinical settings. Responses from the final year medical students in the United Kingdom during the COVID-19 pandemic showed that cancellation, shortened, or postponed student assistantships were significant factors affecting both preparedness and confidence to assist in hospital internships. Cancellation of objective structured clinical examination, cancelled or changed modes of written exams had a significant effect only on their preparedness [25].

\section{Limitation}

As with the majority of studies, this study has some limitations. First, its design was a cross-sectional study without longitudinal data. The findings may not possible to establish a true cause and effect relationship. Although knowing that some factors and happiness were associated, it is impossible to interpret what is the exposure or the outcome. Second, the period of the obtained information was not the actual time of the first outbreak, asking about things that happened in the past could lead to misinformation. All questions that referred to their idea during the first outbreak, had already been highlighted with the word "during the COVID-19 pandemic" to remind the responders. Third, results from a single-medical school survey in Northern Thailand are not possible to generalize to other medical schools with differences of cultural features, curriculums, accessibility of online technology, or existing support systems. These differences are at least different ways to cope with the situation of COVID-19 pandemic, different distance e-learning design or preparedness. Fourth, 25.5\% of the response rate in this study may not reflect the majority of the medical students. The medical student response rate among the cross-sectional studies about COVID-19 was varied $5.9 \%$ of final year students in the United Kingdom [25], 68.3\% of students in Singapore [21], 63.5\% [19] and 67.1\% [20] of students in Turkey, 65.8\% of students in South Korea [23], 82.5\% of students in Jordan [23], and 87\% of students in Saudi Arabia [7]. The authors should improve techniques to recruit more 
responses such as a research fund for the responders with the balance of fulfilling voluntarily exact answers.

\section{Recommendations for future practice}

Based on medical students' responses about the impact of happiness and stress from a single-medical school in Northern Thailand, the author would present some recommendations. First, some demographic characteristics were different between preclinical and clinical students. Data obtained from medical students under different situation awareness or different policy in curriculums such as ward work during the disease outbreak should be separated or compared between preclinical and clinical years. Second, for the application of the results in real situations and direct benefit to the participants, the validity of the responses is required. The students should be encouraged for a higher rate of responses. Third, a study in other expanded geographic and cultural populations should increase the validity of the results.

\section{Conclusion}

There was no difference in the happiness and stress level by the Thai-OHQ scores and Thai-ST5 between preclinical and clinical CMU medical students in Northern Thailand during the first COVID-19 pandemic. To increase happiness, promoting awareness of health satisfaction and regularity of exercise for the medical students should be initiated. To improve student satisfaction if social distancing during a disease pandemic is abruptly required, the preparedness of online and distance learning by the faculty should be continued promptly. To direct the information during a disease outbreak such as the COVID-19 pandemic, Facebook, and Twitter are the primary platforms to use. The decision to continue or suspend the medical student academic schedule, especially for clinical year medical students who would routinely be in a clinical setting during the pandemic, should balance between education goals and student safety in a critical clinical situation. Evidence-based information about factors affecting any aspects of medical students' need while studying in the MD program should be explored. The medical educators and the faculties should carefully select those related factors to achieve the best design for the curriculum modification in a difficult situation.

\section{ORCID:}

Suwicha Kaewsiri Isaradisaikul: https://orcid.org/0000-0003-1332-9940; Pichcha Thansuwonnont: https://orcid.org/0000-0003-3785-4694; Phatsaworn Sangthongluan: https://orcid.org/0000-0003-1597-6121

Acknowledgements: The authors would like to thank Dr. Panomporn Phoomchan (Faculty of Social Sciences, Kasetsart University, Bangkok, Thailand) for suggestion about the Thai-OHQ. The authors would like to thank Dr. Phichayut Phinyo (Department of Family Medicine, and Center for Clinical Epidemiology and Clinical Statistics, Faculty of Medicine, Chiang Mai University, Chiang Mai, Thailand) for assistance in conducting the statistical analysis.

Funding: No financial support was received for this study.

Conflicts of interest: No potential conflict of interest relevant to this article was reported.

Author contributions: SKI contributed to conception and design, analysis and interpretation of data, writing and revising the article; PT contributed to conception and design, acquisition of data, analysis and interpretation of data, drafting the article; and PS contributed to analysis and interpretation of data, reviewing the article. 


\section{References}

1. Holmes EA, O'Connor RC, Perry VH, et al. Multidisciplinary research priorities for the COVID-19 pandemic: a call for action for mental health science. Lancet Psychiatry. 2020;7(6):547-560.

2. Sher L. The impact of the COVID-19 pandemic on suicide rates. QJM. 2020;113(10):707-712.

3. Zhang Y, Ma ZF. Impact of the COVID-19 pandemic on mental health and quality of life among local residents in Liaoning province, China: a cross-sectional study. Int J Environ Res Public Health. 2020;17(7):2381.

4. Srichannil C. The COVID-19 pandemic and Thailand: a psychologist's viewpoint. Psychol Trauma. 2020;12(5): 485-487.

5. World Health Organization. Coronavirus disease (COVID-19) pandemic. https://www.who.int/emergencies/ diseases/novel-coronavirus-2019. Published 2019. Accessed September 5, 2020.

6. Ministry of Foreign Affairs of the Kingdom of Thailand. A nationwide curfew. https://protocol.mfa.go.th/en/content /1 16160-covid-19-update:-a-nationwide-curfew-to-control -the-further-spread-of-the-covid-19-(regulations-no-2)?pa ge $=5 f 3 c 96 f c a 016 b 05 b 47618 \mathrm{ae} 2 \& m e n u=5 f 4 d f d 058588$ dc7f0b603c62. Published 2019. Accessed September 5, 2020.

7. Al-Rabiaah A, Temsah MH, Al-Eyadhy AA, et al. Middle East respiratory syndrome-corona virus (MERS-CoV) associated stress among medical students at a university teaching hospital in Saudi Arabia. J Infect Public Health. 2020;13(5):687-691.

8. Babenko O, Daniels LM, Ross S, White J, Oswald A. Medical student well-being and lifelong learning: a motivational perspective. Educ Health (Abingdon). 2019;32(1):25-32.

9. Kamthan S, Sharma S, Bansal R, et al. Happiness among second year MBBS students and its correlates using Oxford Happiness Questionnaire. J Oral Biol Craniofac Res. 2019;9(2):190-192.

10. Wongratanacheewin J, Vimonvanich S, Teeratakulpisarn $\mathrm{N}$, et al. The level of happiness and possibly related factors among medical students of Khon Kaen University in the 2015 academic year. Srinagarind Med J. 2016; 31(1):112-124.

11. Kulkarni S, Sanjeev C. Happiness level among medical students of a medical college. Int J Community Med Public Health. 2019;6(7):3024-3028.

12. Tan MN, Mevsim V, Pozlu Cifci M, et al. Who is happier among preclinical medical students: the impact of chronotype preference. Chronobiol Int. 2020;37(8): 1163-1172.

13. Yiengprugsawan V, Somboonsook B, Seubsman SA, Sleigh AC. Happiness, mental health, and sociodemographic associations among a national cohort of Thai adults. J Happiness Stud. 2012;13(6):1019-1029.

14. Hills P, Argyle M. The Oxford Happiness Questionnaire: a compact scale for the measurement of psychological well-being. Pers Individ Dif. 2002;33(7):1073-1082.

15. Phoomchan P. Happiness and well-being of Thai agriculturists. J Psychiatr Assoc Thailand. 2015;60(3): 235-248.

16. Silpakit O. Srithanya stress scale. J Ment Health Thailand. 2008;16(3):177-185.

17. Rose S. Medical student education in the time of COVID-19. JAMA. 2020;323(21):2131-2132.

18. Ferrel MN, Ryan JJ. The impact of COVID-19 on medical education. Cureus. 2020;12(3):e7492.

19. Torun F, Torun SD. The psychological impact of the COVID-19 pandemic on medical students in Turkey. Pak J Med Sci. 2020;36(6):1355-1359.

20. Aker S, Midik Ö. The views of medical faculty students in Turkey concerning the COVID-19 pandemic. J Community Health. 2020;45(4):684-688. 
21. Compton S, Sarraf-Yazdi S, Rustandy F, Radha Krishna LK. Medical students' preference for returning to the clinical setting during the COVID-19 pandemic. Med Educ. 2020;54(10):943-950.

22. Gangwar A, Tiwari S, Rawat A, et al. Circadian preference, sleep quality, and health-impairing lifestyles among undergraduates of medical university. Cureus. 2018;10(6):e2856.

23. Park J, Park H, Lim JE, Rhim HC, Lee YM. Medical students' perspectives on recommencing clinical rotations during coronavirus disease 2019 at one institution in
South Korea. Korean J Med Educ. 2020;32(3):223-229.

24. Al-Balas M, Al-Balas HI, Jaber HM, et al. Distance learning in clinical medical education amid COVID-19 pandemic in Jordan: current situation, challenges, and perspectives. BMC Med Educ. 2020;20(1):341.

25. Choi B, Jegatheeswaran L, Minocha A, Alhilani M, Nakhoul M, Mutengesa E. The impact of the COVID-19 pandemic on final year medical students in the United Kingdom: a national survey. BMC Med Educ. 2020; 20(1):206. 